Copyright $@ 2010$ Institute of Electrical and electronics Engineers, Inc.

All Rights reserved.

Personal use of this material, including one hard copy reproduction, is permitted.

Permission to reprint, republish and/or distribute this material in whole or in part for any other purposes must be obtained from the IEEE.

For information on obtaining permission, send an e-mail message to stds-igr@ieee.org.

By choosing to view this document, you agree to all provisions of the copyright laws protecting it.

Individual documents posted on this site may carry slightly different copyright restrictions.

For specific document information, check the copyright notice at the beginning of each document. 


\section{A Robotic Opto-tactile Sensor for Assessing Object Surface Texture}

\author{
A. M. Mazid \\ Faculty of Engineering \\ Monash University \\ Clayton, Victoria 3800, Australia \\ abdul.mazid@eng.monash.edu.au
}

\author{
R. Andrew Russell \\ Intelligent Robotics Research Centre \\ Department of Electrical and Computer Systems \\ Engineering, Monash University, \\ Clayton, Victoria 3800, Australia \\ andy.russell@eng.monash.edu.au
}

\begin{abstract}
This paper presents the design construction and working principle of a newly developed opto-tactile sensor for object surface assessment in robotic applications. The sensor provides information about surface texture and this can be used to assist in quality assurance and object recognition tasks. Surface texture causes flexing of protrusions on the sensor surface which in turn rotates a small mirror within the sensor. This rotation is measured optically as a variation in light intensity transmitted between two optical fibers. A mathematical relationship between these light intensity changes and geometrical parameters of the surface texture has been developed. The sensor has been fabricated and tested on a number of different surface textures and preliminary results of sensor output for differing textures are presented. It is envisaged that the sensor will be integrated into a robot system or other intelligent machine to assist with object recognition and evaluation of surface texture.
\end{abstract}

Keywords - Intelligent grasping, slip detection, tactile sensor, surface texture, mechatronics, opto-tactile sensor, sensor design.

\section{INTRODUCTION}

Tactile sensors, involving arrays of force sensing elements are recognized as a principal requirement for the next generation of intelligent robots [1] employed in service applications. In fact, all robot applications require some form of sensing. Objects can be sensed by vision, hearing, smelling or touching; touch can be sensed by measuring robot internal parameters such as joint torque or external quantities such as skin deflection [2]. Many robot tasks require contact between the robot and its surrounding environment. Such tasks include object manipulation by a robot hand - in an assembly operation, and probing an unknown environment through the robot end-effectors. Physical contact between a robot and its rigid environment requires the robot to be mechanically compliant, in order to avoid excessive contact force. In this kind of situation robot control would involve force sensing to limit contact force. However, for manipulation some minimum force would need to be maintained to avoid slipping of a grasped object. Tactile sensors may detect presence or absence of contact, and more complex tactile sensors may provide data on the shape, size, position, thermal conductivity, and forces applied to a touched object [3]. The tactile sensor considered in this paper can measure some characteristics of the surface texture of objects manipulated by a robot.

Information about the surface texture of an object would hold in identifying the object and could also provide information about the forces necessary to establish a stable grasp. There are wide variations in the surface textures that a robot might encounter such as carpet, floor vinyl, road surface, stone, timber, machined products, and cast items. Therefore, sensing surface texture can provide important information to assist with recognition of objects.

A number of tactile sensors have been developed with the aim of providing the next generation of robots with improved object recognition capabilities. Most of these sensors consist of arrays of taxels covering a large area and attempting to mimic the sensing capabilities of human skin. The opto-tactile sensor developed in this project contains sensing bumps resembling human finger ridges and these are used to assess texture as they are drawn over a surface. The sensor may also be useful for slip detection and for estimating tangential forces during grasping. These possibilities will be the subject of future investigations.

Other researchers have investigated tactile sensors for measuring surface texture and detecting slip. Interest in designing texture and slip sensors first developed amongst scientists and researchers from the beginning of second half of the last century in Japan. One of the first examples was published in the proceedings of the Second International Congress on Industrial Robotics. The authors Ueda, Iwata, and Shingu designed, constructed, and demonstrated their slip detection device [5] that used movement of an object's surface texture past the sensor to detect slip during grasping. In their device, the authors used a sapphire needle to sense the surface texture of slipping objects. The needle was connected to a Rochelle salt crystal that served as vibration transducer. An oscillogram of the crystal output demonstrated the detection of slip during grasping by robot fingers.

Later, Patterson and Nevill [1] developed their induced vibration touch sensor (IVTS) for object surface assessment. The IVTS consisted of a corrugated silicon rubber skin, a 
transduction element, and a mechanism for sliding the object surface in relation to the rubber skin. The prototype sensor was able to recognize the size and shape for characters from the Braille alphabet, the slot on a screw head, and also different grades of sand paper. However, because this sensor employed piezoelectric polymer as sensor element it would be unable to measure steady deflection of the sensor ridges as might occur during object grasping and manipulation.

In their dynamic tactile sensing research, Tremblay and Cutkosky [4] developed an incipient slip-detecting sensor, which was able to assess both normal and tangential grasping forces. This information is important for many applications of

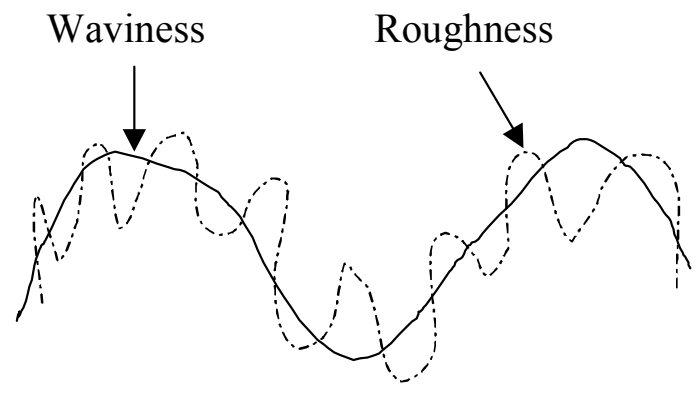

Figure 1. Object surface texture.

robots and intelligent machines that involve manipulation of objects of various sizes, shapes, and masses. Their sensor consisted of a foam core surrounded by a silicon rubber skin with nibs protruding from the outer surface. A number of accelerometers were mounted on the inner side of the rubber skin and supported by the foam core. The slip-detecting concept was based on the principle that, any relative motion between the grasped object and the sensor, produced vibrations through the sudden breaking of contact between the nibs and touched object. These vibrations were detected by the accelerometers and the signals were then further processed. The characteristics of the sensor directly depended on the vibration response of the nib material, which was a highly damping rubber. Object surface profile roughness and irregularities were considered in the sensor design. This design would be possible integrate into a robot gripper used for grasping and handling objects.

It is envisaged that the tactile sensor being developed in this

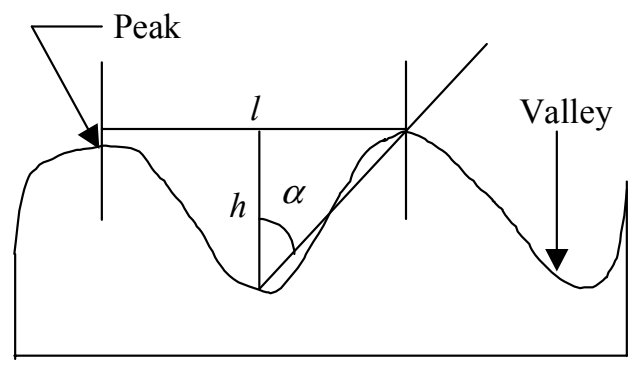

Figure 2. Parameters of surface texture. project will be able to assess surface texture as well as estimating tangential force created in between the grasped object and sensor.

\section{SURFACE TEXTURE OF OBJECTS}

Object surface texture, in general, can be characterized by surface roughness and surface irregularities or waviness as depicted in Fig. 1. The surface profile of most objects can be modeled in this way.

Surface roughness or waviness is characterized by the geometry of peaks and valleys as illustrated in Fig. 2. For a tactile sensor or a stylus, surface texture variation can be assessed [6] by geometric parameters such as rise angle $(\alpha)$, height $(h)$ of one peak from the corresponding valley, and distance $(l)$ from one peak to the next.

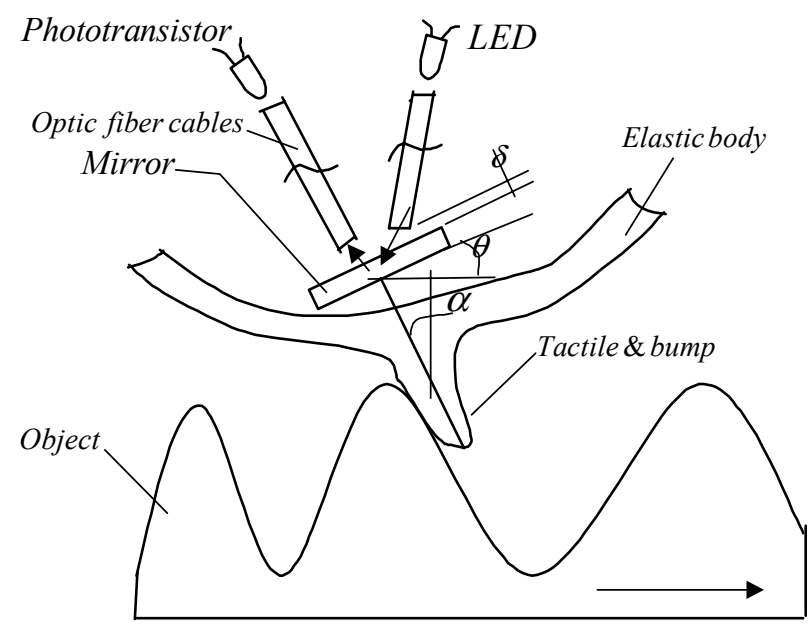

Figure 3. Opto-tactile sensor for object surface recognition.

\section{OPTO-TACTILE SENSOR SYSTEM}

The opto-tactile sensor system was designed and built for assessing object surface texture as an aid to object recognition. The sensor works on the principle of measuring deflection of a bump on the sensor surface by using a small mirror to deflect light traveling between two optical fibers - one acting as a source and the other as a detector. A cross-section view of the sensor is shown in Fig. 3. It consists of the following optical and electronic components:

1. a small piece of full-silvered mirror,

2. the tactile-pin molded into the elastic body of the sensor,

3. a light emitting diode (LED) - model ST23G,

4. a phototransistor of model EL23G, and

5. two lengths of optical fiber cable

The full-silvered mirror is mounted on the inner surface of the sensor skin (body) at a point where its orientation can be affected by deflection of the tactile pin. The tactile pin or bump which extends below the mirror has the same sensing function as the fingerprint ridges of the human hand. The pin and mirror 
can rotate as if hinged at the point where the bump joins the elastic body. Light from a light-emitting diode (LED), is guided down one of the optical fibers to the mirror. Depending on the orientation of the mirror, some portion of the light is reflected towards the second optical fiber where it is directed to the phototransistor. The sensor ends of the optical fibers are both positioned at a distance of $\delta=0.50 \mathrm{~mm}$ from the mirror. The core of the sensor is made of cork and these components are embedded into it to maintain their proper relative positions. The signal from the transistor is digitized by one of the analogue to digital converter (ADC) channels in an Infineon $\mathrm{C} 167$ microprocessor mounted on a PHYTEC KitCON-167 evaluation board. A program written in $\mathrm{C}$ controls the sampling rate of the ADC and downloads it to a PC where further processing is performed using MATLAB ${ }^{\circledR}$ software. The photograph shown in Fig. 4 illustrates the major components of the opto-tactile sensor system.

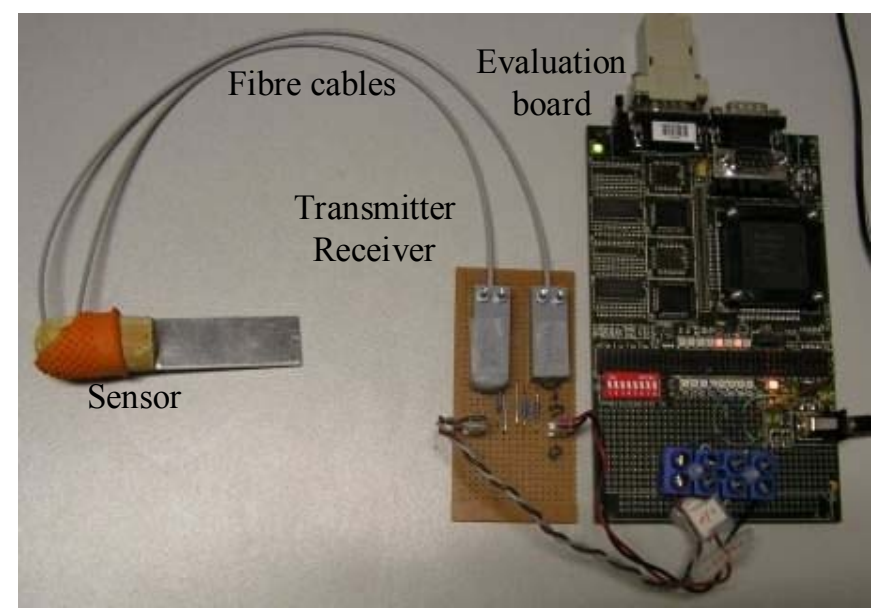

Figure 4. The opto-tactile sensor system.

During motion of the sensor relative to the surface of an object, the tactile pin is deflected by undulations in the object surface. Accordingly, the mirror secured at the top of the tactile pin changes its orientation which in turn changes the intensity $(I)$ of reflected light beam received by the receiver - phototransistor. Thus the intensity of the light beam received by the transistor depends directly on the position of the fullsilvered mirror in relation to the light ray direction. This phenomenon was used to design the sensor for assessing surface texture of different objects manipulated by a robot gripper.

The operating principle of the sensor is simple and with further development it may be possible prepare a sensor suitable for commercial application in robotics areas.

\section{CO-RELATION BETWEEN SURFACE TEXTURE GEOMETRY AND SENSOR OUTPUT}

Intensity of light propagation through the sensor to the phototransistor is proportional to the geometrical parameters of the surface texture, such as height, pitch-distance of the peaks, and angle of rise of individual peaks. This relationship can be expressed as follows:

$$
I=f(l, h, \alpha)
$$

Where,

$I=$ Intensity of reflected light received by the phototransistor from the second fiber optic cable;

$l=$ Pitch distance between two consecutive peaks of surface texture;

$h=$ Height of a peak, from the valley, the stylus is in contact at some moment;

$\alpha=$ Angle of rise of a peak from the valley (Fig. 2).

Considering an object surface is a plane one, there is no deflection of the tactile bump. Therefore, the intensity of the propagated light ray achieves the highest value. Let this peak value be denoted by $I_{\max }$. That means if $\gamma=\theta=0$, then $l=0, \quad h=0$, and $\alpha=0$.

Where, $\gamma=$ angle of inclination of the tactile bump (Fig. 5). The tactile pin is rigidly and perpendicularly attached to the mirror and therefore, it is evident that, $\theta=\gamma$.

Now considering a textured surface as depicted in Fig. 5, where there is at least some deflection of the tactile bump, which changes its angle $\gamma$ depending on the surface texture geometry.

We can assume, in any case the relation between $\gamma$ and $\theta$ is

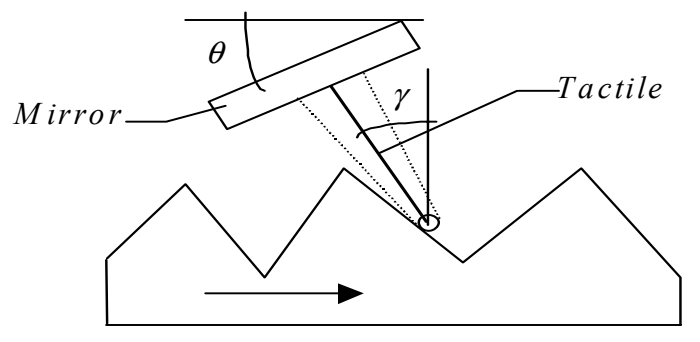

Figure 5. Tactile sensor in motion on an object surface.

expressed as follows:

$$
\gamma=\theta
$$

Now, analyzing the geometry of a single peak and corresponding valley as shown in Fig. 6, we can write,

$$
\tan \alpha=\frac{l}{2 h}
$$

Therefore,

$$
I_{\max } \propto \tan \alpha_{\max }=\frac{l}{2 h}
$$




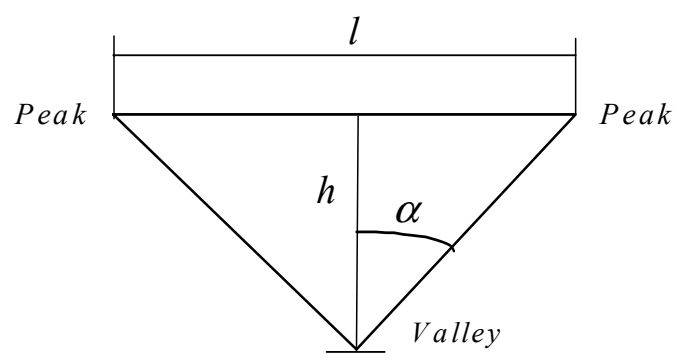

Figure 6. Geometry of a pair of peaks and a valley.

Where, $\alpha_{\max } \leq 90^{\circ}$. Therefore, for general cases, we can express

$$
I \propto \tan \alpha \quad \text { and } \quad I \propto \frac{l}{2 h}
$$

Taking into consideration a coefficient of proportionality, while $l$ and $h$ are variables, (4) can be rewritten as follows:

$$
I=C \frac{l}{2 h}
$$

Or

$$
C=\frac{2 h I}{l}
$$

Where, $C=$ coefficient of intensity for the system.

Using any one of these two equations - (5) and (6) it is possible to define / assess surface texture of any object with the help of the developed sensing mechanism.

\section{A Special Case}

Considering a special case of surface texture as depicted in Fig. 7 , where $h \approx l$.

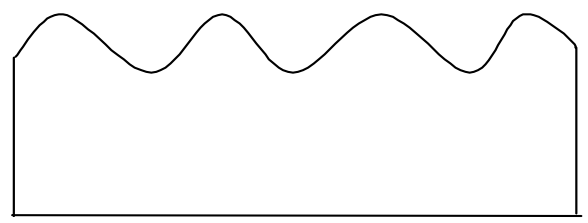

Figure 7. A 'special case' surface texture.

For this case, we can say, $\frac{h}{l} \approx 1$; therefore, (6) takes the following form:

$$
C=2 I
$$

Hence, for a value of $I, C$ can be determined theoretically or, $C$ can be determined by experiment if $I$ is unknown .

For the circuit (Fig. 8), used for the sensor, following Ohm's and Kirchoff's laws, we can write the following equation:

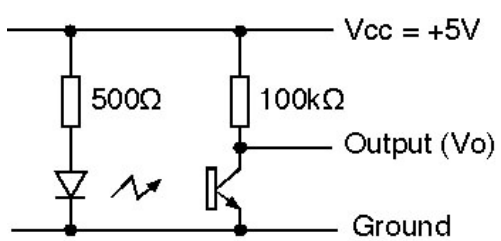

Figure 8. Sensor Circuit.

$$
5 v-(100 \mathrm{k} \Omega) I_{c}=V_{0}
$$

Or,

$$
V_{c c}-R_{c} I_{c}=V_{o}
$$

Where,

$I_{c}=$ Collector current

$R_{c}=$ Collector resistance;

$V_{0}=$ Sensor output voltage;

$V_{c c}=$ Collector voltage

The output voltage $V_{0}$, obtained from the phototransistor after processing, depends on the geometrical parameters of the surface texture such as height $h$, pitch-length $l$ of the peaks, angle of inclination $\alpha$ of individual peaks, and transistor characteristics.

\section{EXPERIMENTS AND RESULTS}

For the first set of experiments the sensor was moved manually instead of using a mechanized system. The sensor hand-held and with light downward pressure it was drawn over the surface in a straight path. During each experiment the output of the sensor was sampled and data values passed to a $\mathrm{PC}$ by the Infineon C167 microprocessor evaluation board (Fig. 4). The data values were imported into MATLAB ${ }^{\circledR}$ which was used to generate time vs. sensor output voltage graphs for number of object surface textures.

In the future it would be more convenient to mount the sensor on a semiautomatic machine for the experiments allowing it to scan an object surface with a constant speed. Alternatively, the object could be moved with constant speed past a stationary sensor. However, the second option may be more difficult to apply if the object under investigation has an uneven profile. The ultimate aim is to assemble this kind of sensor into the fingers of a robot gripper. Here the sensor could be used in the process of object recognition and stable grasping.

In this preliminary investigation, a number of different materials with varying surface characteristics have been tested to demonstrate the capability of the newly developed optotactile sensor. The graphs shown in Fig. 9 represent the sensor output when scanned over a low-pile carpet tile, a laminated plastic tabletop, and a cast iron surface. 


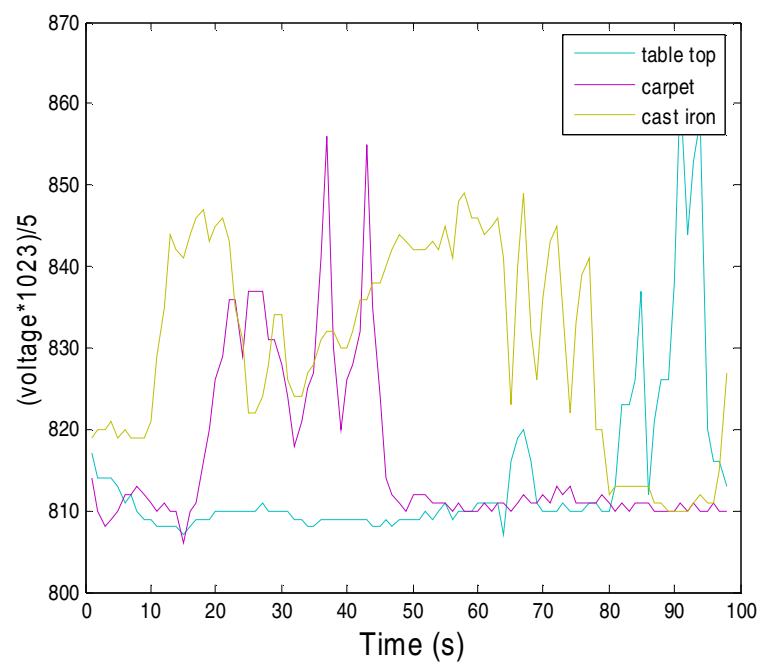

Figure 9. Surface texture detected by the opto-tactile sensor.

The sensor was scanned manually and this would have resulted in some unavoidable variation in the scanning speed. Even allowing for this it is evident that the graphs do capture some of the surface characteristics of the different surfaces. The relatively smooth tabletop produced an output voltage with less variation than that of carpet and the cast iron surfaces. Regular tufts of carpet fibers resulted in a regular periodic graph; and the cast iron surface is characterized as an unpredictably textured surface. Scans of other surfaces, which are not presented in this article, have also produced sensor data that corresponds to the type of surface.

The preliminary results indicate that the sensor can provide useful data relating to surface texture.

\section{CONCLUSION AND DISCUSSION}

Considering the performances of the opto-tactile sensor and the experimental results the following conclusion can be derived:

$\diamond \quad$ A new tactile sensing technique has been proposed that has potential uses in robotic technology and intelligent mechatronic machine design.

$\diamond \quad$ A mathematical model has been developed relating the sensor output voltage to the geometrical parameters of peaks and valleys, and angle of inclination of peaks of surface roughness and irregularities.

$\diamond \quad$ It is expected that using the opto-tactile sensor, it will be possible to sense tangential, as well as, normal forces applied by grippers during grasping operations.

$\diamond \quad$ The sensor is relatively simple to manufacture and uses inexpensive components such as the rubber skin with bumps on one side, the mirror, optical fiber cables, an
LED, and a phototransistor, together with means for signal processing and surface recognition.

$\diamond \quad$ The sensing capabilities of the opto-tactile sensor are governed to some extent by the diameter of the end of the tactile pin. This may limit the range of scales of textures that can be sensed. However, for some purposes it may be enough to recognize that the particular surface is fairly smooth.

$\diamond \quad$ A further continuation pathway of this work has been indicated.

The opto-tactile sensor concept provides opportunities for further work in the areas of object recognition and grasping for robotic handling of different types of materials. It is also envisaged that the sensor will be useful for measuring tangential forces during grasping and this possibility will be investigated further.

\section{REFERENCES}

[1] Robert W. Patterson and Gale E. Nevill, Jr., "The induced vibration touch sensor - a new dynamic touch sensing concept," Robotica, vol. 4, pp. 27-31, 1985.

[2] R. Andrew Russell, Robot Tactile Sensing. Prentice Hall. 1990.

[3] Howard R Nicholls (ed), Advanced Tactile Sensing for Robotics. World Scientific. London. 1992.

[4] Marc R. Tremblay, Mark R. Cutkosky, "Estimating friction using incipient slip sensing during a manipulation task," in Proc. IEEE International Conference on Robotics and Automation, vol. 1. Atlanta, Georgia, USA, 1993, pp. 429-434.

[5] Minora Ueda, Kazuhide Iwata, Hiroyashu Shingu, "Tactile sensor for industrial robot to detect a slip," in Proc. Second International Symposium on Industrial Robotics, 1972, USA. pp. 63-76.

[6] M. A. Mazid, N. Zhang, M. Alauddin, O. N. Trifonov, Theoretical basis for assessment of machine tools by scattered energy of vibration. 1998 Pacific Conference on Manufacturing (PCM 98). Brisbane, Australia. 1998, pp. 933-938. 